\title{
Subfunctionalization of a Retinoid-Binding Protein Provides Evidence for Two Parallel Visual Cycles in the Cone- Dominant Zebrafish Retina
}

\author{
Valerie C. Fleisch, ${ }^{1,2 *}$ Helia B. Schonthaler, ${ }^{2 *}$ Johannes von Lintig, ${ }^{3}$ and Stephan C. F. Neuhauss ${ }^{1,2}$ \\ ${ }^{1}$ Institute of Zoology, ${ }^{2}$ Swiss Federal Institute of Technology (ETH) Zurich, Department of Biology, and Brain Research Institute, University of Zurich, CH- \\ 8057 Zurich, Switzerland, and 3Department of Pharmacology, Case School of Medicine, Case Western Reserve University, Cleveland, Ohio 44106-4965
}

In vertebrates, the absorption of a photon results in an 11-cis to all-trans isomerization of the retinylidene chromophore of cone and rod visual pigments. To sustain vision, metabolic pathways (visual cycles) have evolved that recycle all-trans-retinal back to 11-cis-retinal. The canonical visual cycle takes place in photoreceptor cells and the adjacent retinal pigment epithelium (RPE). Biochemical analyses provided evidence for the existence of an additional cone-specific visual cycle involving Müller glia cells, but none of its molecular components has yet been identified. Here we took advantage of the zebrafish retina to investigate the role of the cellular retinaldehydebinding protein CRALBP in this process. We found that the zebrafish genome encodes two cralbp paralogs: cralbp $a$ and $c r a l b p b$. These paralogs are differentially expressed in the retina. Cralbp a is exclusively expressed in the RPE, and Cralbp b is localized to Müller cells. We used an antisense morpholino approach to knock down each cralbp paralog. Analysis of 11-cis-retinal levels revealed that visual chromophore regeneration is diminished under both conditions. Visual performance, as assessed by electroretinography, revealed reduced light sensitivity in both Cralbp a- and Cralbp b-deficient larvae, but it was more pronounced in Cralbp b-deficient larvae. Cralbp b-deficient larvae also exhibited significant deficits in their visual behavior. Together, these data demonstrate that Cralbp expression in Müller cells is essential for cone vision, thereby providing evidence that both the canonical and the alternative visual cycle depend on the same type of retinoid-binding protein.

Key words: retina; zebrafish; photoreceptors; CRALBP/rlbp1; vitamin A; visual cycle

\section{Introduction}

In vertebrate photoreceptors, absorbance of light by the visual pigments results in an 11-cis to an all-trans isomerization of the visual chromophore (11-cis-retinal) and the initiation of the phototransduction signal cascade (Wald, 1968; Stryer, 1986; Schoenlein et al., 1991; McBee et al., 2001; Palczewski et al., 2006). To sustain vision, 11-cis-retinal must be recycled in a complex sequence of enzymatic reactions, collectively named the visual cycle. The canonical visual cycle takes place in two separate compartments of the retina. In the photoreceptor outer segments, all-trans-retinal is converted to all-trans-retinol. Subsequently, all-trans-retinol is transported to the second compartment, the retinal pigment epithelium (RPE) and esterified into retinyl es-

Received Dec. 19, 2007; accepted June 21, 2008.

This work was supported by the European Commission as part of the ZF-MODELS (Zebrafish Models for Human Development and Disease) Integrated Project in the 6th Framework Programme LSHG-CT-2003-503496 (S.C.F.N., V.C.F.), the Swiss National Science Foundation, and by an internal grant from Case Western Reserve University to J.v.L. We thank Beate Ziser for technical assistance with HPLC analysis and Dr. Oliver Biehlmaier for help with confocal microscopy.

${ }^{*} V$.C.F. and H.B.S. contributed equally to this work

Correspondence should be addressed to Prof. Dr. Stephan C. F. Neuhauss, Institute of Zoology, University of Zurich, Winterthurerstrasse 190, CH-8057 Zurich, Switzerland. E-mail: stephan.neuhauss@zool.uzh.ch.

H. B. Schonthaler's present address: Cancer Cell Biology Program, Spanish National Cancer Research Centre, E-28029 Madrid, Spain.

DOI:10.1523/JNEUROSCI.2367-08.2008

Copyright $\odot 2008$ Society for Neuroscience $\quad$ 0270-6474/08/288208-09\$15.00/0 ters (REs). REs are then converted to 11-cis-retinol by the action of a retinoid isomerase encoded by the Rpe65 gene (Jin et al., 2005; Moiseyev et al., 2005; Redmond et al., 2005). Finally, 11cis-retinol is oxidized to 11-cis-retinal and shuttled back to the photoreceptor outer segment. The reactions in the RPE depend on cellular retinaldehyde-binding protein (CRALBP), which acts as a substrate carrier to chaperone retinaldehyde through an aqueous milieu (Winston and Rando, 1998; Stecher et al., 1999). Mice with an inactivated Cralbp gene exhibit impaired visual pigment regeneration. Both 11-cis-retinal production and dark adaptation after illumination are delayed and coincide with an accumulation of all-trans-retinyl esters, consistent with a role in the canonical visual cycle (Saari et al., 2001).

The expression of CRALBP in Müller glia cells of the inner retina suggests an additional function of this protein (BuntMilam and Saari, 1983; Saari and Bredberg, 1987). One possibility might be that CRALBP expressed in Müller glia cells participates in cone visual pigment regeneration. Recent biochemical studies with cone-dominated retinas isolated from chicken and ground squirrel provided evidence for such an alternative visual cycle for cones (Mata et al., 2002, 2005; Muniz et al., 2007). These biochemical data are supported by in vivo studies in zebrafish (Danio rerio) larvae, showing that the loss of Rpe65, the retinoid isomerase of the canonical visual cycle, only marginally affects cone vision (Schonthaler et al., 2007). 
Thus far, none of the molecular components of this putative alternative visual cycle has been molecularly identified. In the present study, we show that the genome of zebrafish encodes two paralogs of cralbp (cralbp $a$ and $b$ ), which show spatially nonoverlapping expression: cralbp $a$ is expressed in the RPE and cralb $b$ is expressed in Müller glia cells. This unique genetic constellation allows the analysis of the function of Cralbp either in the RPE or Müller cells. To approach this, we used an antisense morpholino approach to knock down either one or both proteins. In respective Cralbp a- and Cralbp b-deficient larvae, we functionally tested visual performance by electroretinography (ERG) and visual behavior analysis and additionally performed HPLC profiling for 11-cisretinal. Together, our data demonstrate that Cralbp expression in both Müller cells and the RPE is required for cone visual pigment regeneration.

\section{Materials and Methods}

Fish care and strains. Zebrafish of the Tü strain were used as the wild-type strain (Dahm et al., 2005). Zebrafish were maintained at $28^{\circ} \mathrm{C}$ under a 14/10 h light/dark cycle and bred and crossed as described previously (Westerfield, 1994). Larvae were raised in E3 medium [containing (in mM) $5 \mathrm{NaCl}, 0.17 \mathrm{KCl}, 0.33 \mathrm{CaCl}_{2}$, and $0.33 \mathrm{MgSO}_{4}$ ] until $5 \mathrm{~d}$ postfertilization (dpf).

Cloning. Putative zebrafish cralbp genes were identified via a homology search of the Zv7 Ensembl database (http://www.ensembl.org/ Danio_rerio) using the human CRALBP protein sequence as query. Total RNA was isolated from $5 \mathrm{dpf}$ zebrafish larvae using the RNAeasy kit (QIAGEN) according to the manufacturer's instructions. cDNA was synthesized using the SuperScript III Reverse Transcriptase Kit (Invitrogen) as described in the manual. To amplify the coding sequence from the cDNA, the following primers were used. cralbp a: sense, AACGTTGCATTGAGTATTTGAAGC; antisense, GAGACTTATCCAGTTTCGCTTGG. cralbp b: sense, TGGAATAGTTGACGGTTCATTCC; antisense, AAAAGGGCTGTGTCTTCAGATC.

To obtain $5^{\prime}$ and $3^{\prime}$ sequences of the cralbp cDNA, RACE-PCR was performed with the SMART RACE cDNA Amplification Kit (Clontech). PCR products were cloned into the pCRII-Vector (Clontech) and sequenced with T7 and SP6 primers (GenBank accession number for cralbp $a$, EU348854, and for cralbp b, EU348853).

In situ hybridization (whole mount and slides). Sense and antisense probes for in situ hybridization (ISH) were transcribed in vitro from a linearized pCRII-Vector containing the full-length cralbp $a$ and $b$ coding sequence using SP6 and T7 polymerases, respectively, in the presence of digoxigenin-coupled nucleotides (DIG RNA Labeling Kit; Roche Molecular Biochemicals). Transcripts were hydrolyzed to obtain fragments of $\sim 300-500 \mathrm{nt}$ length. To prevent melanization of skin melanocytes and the RPE, embryos used for ISH were treated with $0.2 \mathrm{~mm}$ 1-phenyl-2thiourea (PTU; Sigma). PTU-treated embryos were staged, fixed in $4 \%$ paraformaldehyde (PFA) in $1 \times \mathrm{PBS}, \mathrm{pH} 7.3$, at $4^{\circ} \mathrm{C}$ overnight, dehydrated in a standard methanol series, and stored in 100\% methanol at $-20^{\circ} \mathrm{C}$. ISH was performed using an automated ISH apparatus (Hölle \&
Hüttner). Hybridization with probes was performed overnight at $62^{\circ} \mathrm{C}$. Detection was performed with an alkaline phosphatase-coupled antidigoxigenin Fab fragment (Roche) and NBT/BCIP (nitroblue tetrazolium/5-bromo-4-chloro-indolyl phosphate) staining buffer. Stained embryos were cleared with 50\% methanol $/ 50 \%$ glycerol and transferred into $100 \%$ glycerol for imaging.

Standard histology. Larvae (5 dpf) were fixed for $1 \mathrm{~h}$ in $4 \%$ PFA in $1 \times$ PBS $\left(0.8 \% \mathrm{NaCl}, 0.02 \% \mathrm{KCl}, 0.02 \mathrm{M} \mathrm{PO}_{4}, \mathrm{pH} 7.3\right)$. After two washing steps with $1 \times$ PBS, they were dehydrated through a series of dehydration steps ranging from $50 \%$ to $100 \%$ ethanol. For preinfiltration, larvae were incubated 1:1 in 100\% ethanol and Solution A [ $25 \mathrm{ml}$ of Technovit 7100 (Heraeus Kulzer) plus $0.25 \mathrm{~g}$ of Hardener 1] two times for $30 \mathrm{~min}$ each. For infiltration, larvae were incubated in $100 \%$ Solution A at $4^{\circ} \mathrm{C}$ overnight. For polymerization, Solution A was mixed with Hardener II (15: 1 ), and finally, larvae were embedded in the polymerization solution.

Sections $(3-5 \mu \mathrm{m})$ were cut using a rotation microtome. Slides were dried, stained with Richardson (Bromeis) solution (1\% methylene blue, $1 \%$ Borax, 1\% azur II, in a ratio of 1:1:2), and covered in Entellan (Merck).

Immunostaining. Wild-type and morpholino-injected larvae were raised in E3 medium containing 0.2 mM PTU to prevent development of pigment in the RPE. 

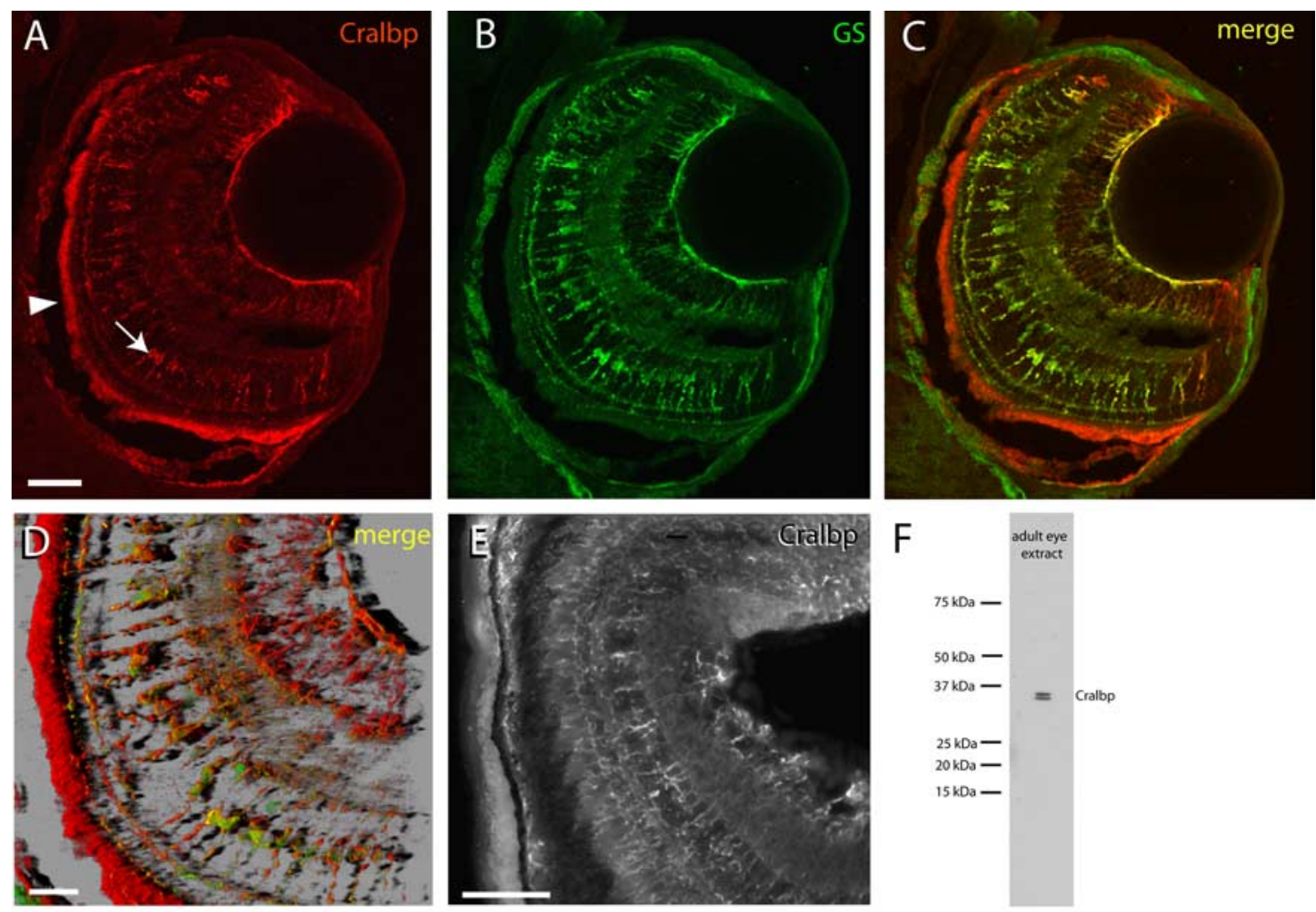

Figure 2. CRALBP antiserum detects both zebrafish Cralbp proteins on immunostaining. $A$, Control ( $5 \mathrm{dpf}$; wild-type) larval sections; the arrowhead points to RPE, and the arrow points to Müller glia cell bodies. $\boldsymbol{B}-\boldsymbol{D}$, Colocalization of Cralbp staining with glutamine syntethase staining. $\boldsymbol{B}$, Immunostaining for glutamine synthetase in radial section of $5 \mathrm{dpf}$ wild-type larvae. $\boldsymbol{C}$, Merge picture of $\boldsymbol{A}$ and $\boldsymbol{B}$. $\boldsymbol{D}$, Higher magnification of double staining using the CRALBP and GS antisera. $\boldsymbol{E}$, Immunostaining of adult section using the CRALBP antiserum. The arrowhead points to RPE, and the arrow points to Müller glia cell bodies. Scale bar: $\boldsymbol{A}, \boldsymbol{E}, 30 \mu \mathrm{m} ; \mathbf{D}, 15 \mu \mathrm{m}$. $\boldsymbol{F}$, Western blot performed on protein extract of adult zebrafish eyes, incubated with the Cralbp antiserum (generously provided by J. Saari).

For immunohistochemistry, larvae were fixed for 45 min in $4 \%$ paraformaldehyde in $1 \times$ PBS, washed with $1 \times$ PBS, and incubated overnight in $30 \%$ sucrose at $4^{\circ} \mathrm{C}$. Fixed larvae were embedded in Tissue Tec medium (Sakura Finetek Europe) and immediately frozen in liquid nitrogen. Sections of $16 \mu \mathrm{m}$ were cut using a cryotome, dried for $30 \mathrm{~min}$ at room temperature, and stored at $-80^{\circ} \mathrm{C}$.

Before using the slides for immunostaining, they were dried for $1 \mathrm{~h}$ at $37^{\circ} \mathrm{C}$, washed in $1 \times \mathrm{PBS}$, and incubated for $4 \mathrm{~h}$ with blocking solution ( $10 \%$ normal goat serum, $1 \%$ bovine serum albumin in $1 \times$ PBS containing $0.3 \%$ Tween). Subsequently, sections were incubated with the primary antibody at $4^{\circ} \mathrm{C}$ overnight, washed two times with $1 \times \mathrm{PBS}$, and incubated with the secondary antibody for $1-2 \mathrm{~h}$ at room temperature. Finally, slides were washed two times with $1 \times$ PBS, covered with Kaisers Glyceringelatin (Merck), and stored at $-20^{\circ} \mathrm{C}$.

For Cralbp immunostaining, CRALBP antiserum (kindly provided by Dr. Johnathan Saari, University of Washington, Seattle, WA) was used at a 1:500 dilution. For double staining with glutamine synthetase (GS), the anti-GS antibody (Millipore Bioscience Research Reagents) was used in a 1:1000 dilution. For immunostaining for rods, the 1D1 antibody (kindly provided by Ann Morris, Florida State University, Tallahassee, FL) was used 1:100, and staining for cones was performed by using the Zpr1 antibody (Zebrafish International Resource Center) in a 1:20 dilution. Alexa Fluor 488 and Alexa Fluor 568 (Invitrogen) directed against rabbit (for Cralbp) or mouse (for all of the other antibodies) were used as secondary antibodies.

Antisense morpholino knock-down. Specific morpholino nucleotides directed against zebrafish cralbp a (GAAATGTTCCACTAACAACCGCCAT) and cralbp $b$ (GCCATAGTGCTCAGATTCTCCACGG) as well as a control were ordered from Gene Tools. Morpholinos were diluted in $1 \times$ Danieau's solution [ $58 \mathrm{~mm} \mathrm{NaCl}, 0.7 \mathrm{~mm} \mathrm{KCl}, 0.4 \mathrm{~mm} \mathrm{MgSO}_{4}, 0.6 \mathrm{~mm}$ $\mathrm{Ca}\left(\mathrm{NO}_{3}\right), 5 \mathrm{~mm}$ HEPES] containing $0.2 \%$ phenol red (for color indication purposes) to the desired concentration. Diluted morpholinos were injected into the 1-2 cell-stage embryo.
Quantification of mRNA levels by real-time PCR. Total RNA from morpholino-injected larvae was isolated using TRIzol (Invitrogen). The isolated RNA was used to generate cDNA by using Ready-To-Go YouPrime First-Strand Beads (GE Healthcare), following the manufacturer's protocol. For the real-time PCR (RT-PCR) analysis, two different primer pairs spanning an exon boundary for each cralbp $a$ and $b$ gene were designed: cralbp $a$, sense: TGAGCTTGCTAAAGGTGTTCAGG and GTCAAACCCCTGATGAAGAGC; antisense: TCAGGATAATCCCGTCTGAAGC and GTGATCTTGCCGTCATATTTGG. cralbp $b$, sense: TTGAACATCTGACTGTGAAAGACC and CTGCGTGCCTACTGTGTAATCC; antisense: GCCTGCCTTGTCTTTAATCATGG and ATCACATGCACAGCCTTAAACC. gapdh (glyceraldehyde-3-phosphate dehydrogenase), sense: CACAACCAAATCAGGCATAATGG; antisense: CATGTAATCAAGGTCAATGAATGG.

Real-time PCR was performed with SYBR Green on an Opticon 2 monitor fluorescence thermocycler (MJ Research). Loading was normalized to gapdh expression, and results were normalized to control larvae.

Photography. Immunostaining and histological sections were photographed under an Olympus BX61 microscope, and pictures were taken with the F-View camera (Olympus). Images were edited using the Olympus Cell Soft Imaging Software.

Pictures of the cralbp $a$ and $b$ expression analysis were taken using an Axioscope 2Mot (Zeiss) connected to an AxioCamHR color camera using the AxioVision 3.0 software (Zeiss).

Optokinetic response measurements. Larval OKR responses were measured as described previously (Rinner et al., 2005a). Single larvae were placed upright in the center of a $35 \mathrm{~mm}$ Petri dish containing $3 \%$ prewarmed $\left(28^{\circ} \mathrm{C}\right)$ methylcellulose for immobilization. Moving square gratings of different contrasts were projected onto a screen by a Proxima 4200 DLP projector. Eye movements triggered by the visual stimulation were recorded by means of an infrared-sensitive CCD camera. Customdeveloped software on the basis of LabView IMAQ (National Instru- 

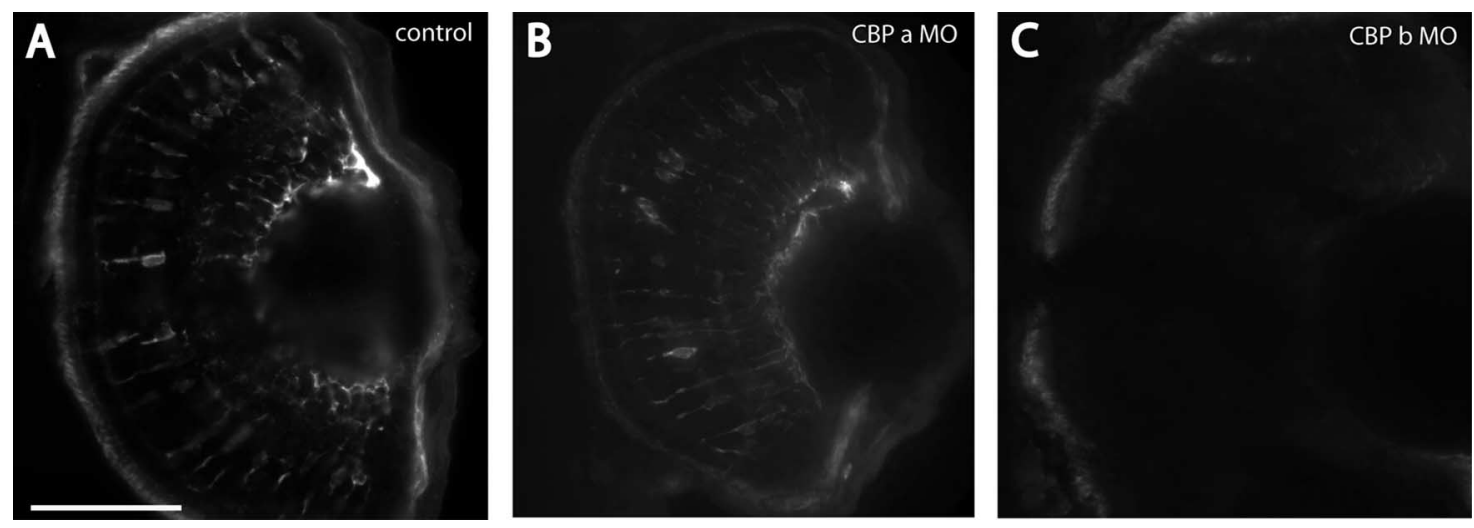

Figure 3. Injection of morpholino oligonucleotides results in reduction of Cralbp a and b protein levels. A, Immunostaining using the CRALBP antiserum on $5 \mathrm{dpf}$ control (wild-type) larval section. B, Immunostaining of section taken from $5 \mathrm{dpf}$ cralbp a morpholino-injected (1 mM) larva. C, Immunostaining of $5 \mathrm{dpf}$ cralbp $b$ morpholino-injected (700 $\mu \mathrm{m})$ larva. Scale bar, $50 \mu \mathrm{m}$.

Table 1. Relative expression levels of cralbp $a$ and $b$ in cralbp $a(\mathrm{MO} a), b$ (MOb), and double (MOa/b) morpholino-injected larvae normalized to controls

\begin{tabular}{lll}
\hline RNA samples & $\begin{array}{l}\text { Relative expression of } \\
\text { cralbp } a \text { to controls }\end{array}$ & $\begin{array}{l}\text { Relative expression of } \\
\text { cralbp } b \text { to controls }\end{array}$ \\
\hline M0 $a$ & $0.22( \pm 0.17)$ & $1.51( \pm 3.59)$ \\
MOb & $0.71( \pm 1.02)$ & $0.29( \pm 0.21)$ \\
M0a/b & $0.29( \pm 0.15)$ & $0.28( \pm 0.23)$ \\
\hline
\end{tabular}

Data are shown as means; $\mathrm{SD}$ is depicted in parentheses.

ments, version 5.1) was used to control stimulation and camera, and to analyze the resulting images.

Statistical analysis was performed using a bootstrap resampling test of pooled data. A detailed description of this statistical method is provided (Rinner et al., 2005b). The significance level has been chosen as $5 \%$ for all measurements.

ERG recordings. Recordings were done as described previously (Makhankov et al., 2004). Larvae (5 dpf) were dark-adapted for at least 30 min before starting the measurements. Larvae were anaesthesized with a drop of $0.5 \% 3$-aminobenzic acid ethyl ester, in $\mathrm{H}_{2} \mathrm{O}, \mathrm{pH} 7$ and positioned right above the reference electrode. The recording electrode was positioned onto the cornea, without damaging the eye. A light stimulus of $100 \mathrm{~ms}$ duration and light intensity of 6000 lux was used to elicit a saturating response. Varying light intensities of $5 \log$ units (log -4 to $\log$ 0 ; $\log 0$ corresponding to $6300 \operatorname{lux}$ ) were used for measuring the intensity-response function. The response of each single larva was measured five times and then averaged. Statistical analysis was done using GraphPad Prism 4 (GraphPad Software) and graphs were drawn using Origin version 7 (OriginLab).

Retinoid analysis. Zebrafish larvae (4 dpf) were dark-adapted overnight. Before illumination, $5 \mathrm{dpf}$ larvae were placed into Petri dishes under dim red light. The bottom and the sides of these dishes were coated with light-reflecting aluminum foil. The larvae were then illuminated for 20 min with 10,000 lux using a KL 1500-T electronic fiber optic device (Schott). On illumination, larvae were dark-readapted for $45 \mathrm{~min}$, then immediately collected under dim red light, killed on ice, and stored at $-70^{\circ} \mathrm{C}$. Retinoids were extracted under monochromatic light $(695 \mathrm{~nm})$ and subjected to HPLC separation as described previously (Schonthaler et al., 2007). For quantification of the molar amounts of retinoids, peak integrals were scaled with defined amounts of reference substances and were quantified using 32 Karat software (Beckman Instruments) as described previously (von Lintig and Vogt, 2000).

\section{Results}

\section{The zebrafish genome harbors two cralbp orthologs}

The cone-dominant retina of the zebrafish is ideally suited for the genetic analysis of photoreceptor function (Rinner et al., 2005a; Schonthaler et al., 2007). We used a combination of bioinformatics and cDNA cloning strategies to isolate genes coding for cralbp.
We identified two paralogs of mammalian Cralbp and denoted them as cralbp $a$ and cralb $b$. By BLAST (basic local alignment search tool) analysis and sequence alignments using the Zv7 assembly of the zebrafish genome (http://www.ensembl.org/ Danio_rerio/index.html), the two cloned genes were located to two different chromosomes, cralbp a to chromosome 25 and cralbp $b$ to chromosome 7 . Both share a high degree of identity to the mouse and human orthologs at the level of the deduced amino acid sequences. Phylogenetic analysis (ClustalW, MegAlign; DNAStar) confirmed that these paralogs are true orthologs to the mammalian Cralbp gene (supplemental Fig. 3, available at www.jneurosci.org as supplemental material).

In linear alignments, these two paralogs share an identity of $\sim 76 \%$ at the nucleic acid and $80 \%$ at the amino acid level (supplemental Figs. 1-3, available at www.jneurosci.org as supplemental material).

\section{Zebrafish cralbp paralogs show complementary retinal expression}

To determine the developmental expression of these genes, we performed whole-mount in situ hybridization experiments with embryos and larvae of different developmental stages. Both cralbp $a$ and cralbp $b$ mRNA expression first became detectable in the pineal gland at $24 \mathrm{~h}$ postfertilization (hpf) (Fig. 1A,B). The pineal is a photosensitive organ in the dorsal diencephalon. Expression in the pineal gland of both cralbp paralogs persisted when development proceeded and was maintained into adulthood.

At $48 \mathrm{hpf}$, the expression of the two cralbp paralogs also became detectable in the retina but was strikingly complementary. Cralbp a was expressed in the developing RPE and cralbp $b$ was expressed in the choroid fissure (Fig. $1 C-F$ ). At later developmental stages, the expression of cralbp a spread out through the RPE (Fig. $1 C, D$ ) and cralbp $b$ was specifically detected in Müller glia cells of the inner retina (Fig. $1 G, H$ ). This expression pattern was preserved in all developmental stages examined and suggests a subfunctionalization of the two Cralbp proteins to different compartments in the zebrafish retina.

To confirm these results on the protein level, we used an antibody raised against mammalian CRALBP (generously provided by J. Saari), which detected two bands corresponding to the two zebrafish Cralbp proteins on a Western blot (Fig. $2 F$ ), and labeled Müller glia cells and RPE cells in the zebrafish retina just as in the mouse retina (Fig. 2A,C-E). This staining pattern was consistent with mRNA expression (see above), suggesting that the antibody 

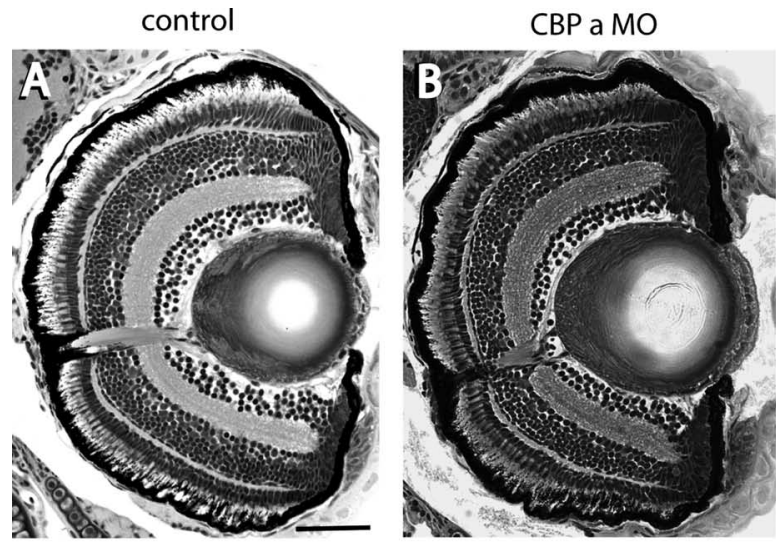

E
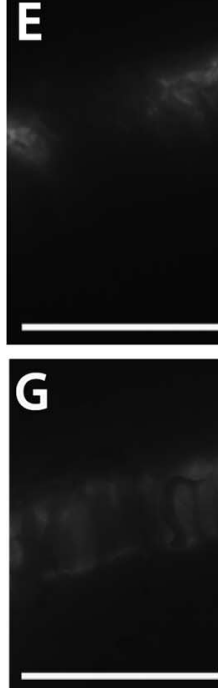

1D1

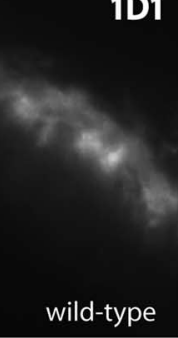

Zpr1
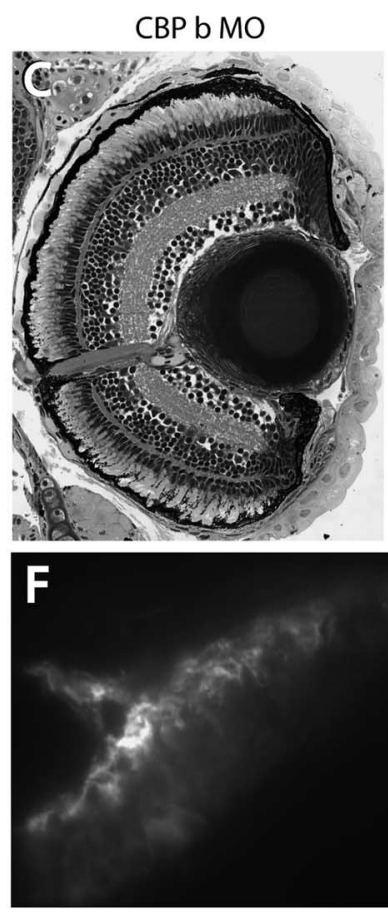

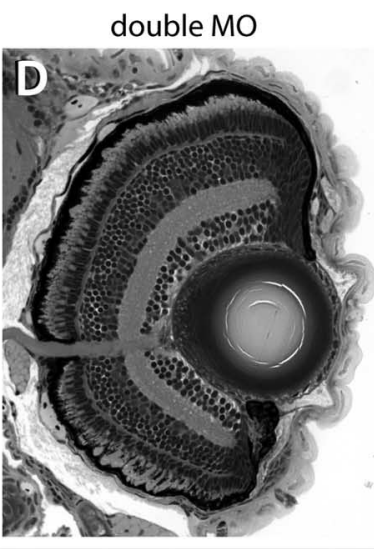

1D1

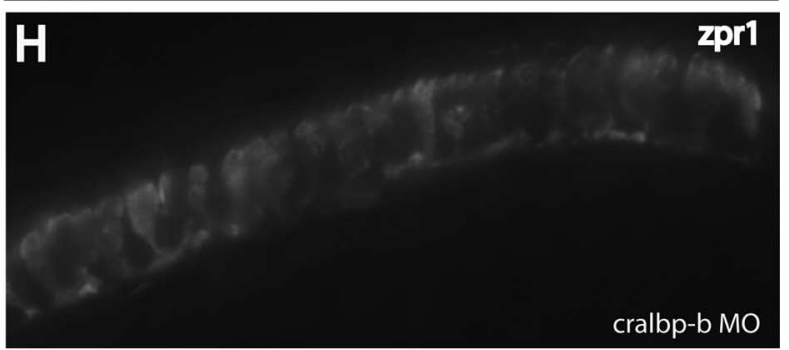

Figure 4. Assessment of retinal and photoreceptor integrity in cralbp $a, b$, and double-morpholino-injected larvae. $\boldsymbol{A}-\boldsymbol{D}$, Radial sections of $5 \mathrm{dpf}$ control and morpholino-injected larvae, stained with Richardson (Bromeis) solution. $\boldsymbol{A}$, Control (wild-type); $\boldsymbol{B}$, cralbp a morpholino-injected larva (1 mM); $\boldsymbol{C}$, cralbp b morpholino-injected larva (700 $\mu \mathrm{m}$ ); $\boldsymbol{D}$, coinjected larva (cralbp a, $800 \mu \mathrm{m}$; (ralbp $b, 500 \mu \mathrm{m}) . \boldsymbol{E}-\boldsymbol{H}$, Immunostaining for rod and cone outer segments using $1 \mathrm{D} 1$ (rod-specific) $(\boldsymbol{E}, \boldsymbol{F})$ and zpr1 (cone-specific) $(\boldsymbol{G}, \boldsymbol{H})$ antibodies. $\boldsymbol{E}$, Wild-type retina stained with $1 \mathrm{D} 1 ; \boldsymbol{F}$, retina of cralbp $a$ morpholino-injected larva (1 mm) stained with $1 \mathrm{D1} ; \boldsymbol{G}$, wild-type retina stained with zpr $1 ; \boldsymbol{H}$, retina of cralbp $b$ morpholino-injected larva (700 $\mu \mathrm{m}$ ) stained with zpr1. Scale bars, $50 \mu \mathrm{m}$.

specifically recognizes both cralbp paralogs in the larval and the adult zebrafish retina.

To confirm that the cells labeled in the inner retina were Müller glia cells, we performed double labeling experiments with the Müller glia cell-specific marker glutamine synthetase (anti-GS). Indeed, we found that Cralbp b and GS are colocalized, thus demonstrating that the Cralbp b protein is expressed in Müller glia cells (Fig. $2 B-D$ ).

\section{Generation of Cralbp deficient larvae}

The complementary expression patterns of the two cralbp genes allowed us to study the function of the two paralogs separately by loss-of-function studies. Toward this end, we designed antisense morpholino nucleotides (MOs) targeted against the translational start sites of either cralbp a or cralbp $b$. This approach is routinely used to specifically knock down gene functions in different embryonic compartments (Nasevicius and Ekker, 2000; Rinner et al., 2005a; Schonthaler et al., 2007). Injection of both cralbp MOs did not cause any developmental malformations. Injected larvae are indistinguishable from mock or control injected larvae throughout development.

Immunohistological staining with the CRALBP antiserum showed that expression of the proteins was highly diminished or absent in the larval retina at $5 \mathrm{dpf}$. Injection of the MO targeted against cralbp a (cralbp aMO) highly diminished labeling for Cralbp in the RPE but did not affected Cralbp protein levels in Müller glia cells (Fig. $3 B$ ). In contrast, injection of morpholino targeting cralbp $b$ (cralbp $b M O$ ) led to a highly reduced staining for Cralbp in the inner retina but did not affect staining for Cralbp in the RPE (Fig. 3C).

The CRALBP antiserum recognizes two bands of the predicted molecular weight for both Cralbp proteins on a Western blot (Fig. 2 F). Unfortunately, in Western blot analysis, Cralbp protein levels were below the detection limit in protein extracts of 5 dpf larvae. Hence, we decided to take an alternative approach using quantitative real-time PCR analysis on reverse-transcribed cDNA to get an indirect estimation of the remaining Cralbp protein levels in larvae that had been injected with morpholinos against cralbp $a, b$, or both.

We observed a reduction of $\sim 78 \%$ of cralbp a mRNA in cralbp a morpholino-injected larvae and $\sim 70 \%$ in double-morpholinoinjected larvae. Similarly, a reduction of $\sim 70 \%$ of cralbp $b$ mRNA in cralbp $b$ and double-injected larvae was detected (Table 1). Interestingly, we also saw an increase of cralbp $b$ mRNA in cralbp a morpholino-injected larvae and vice versa, which was highly variable and might be caused by a compensatory mechanism (Table 1).

Hence, we can manipulate protein expression of the two 

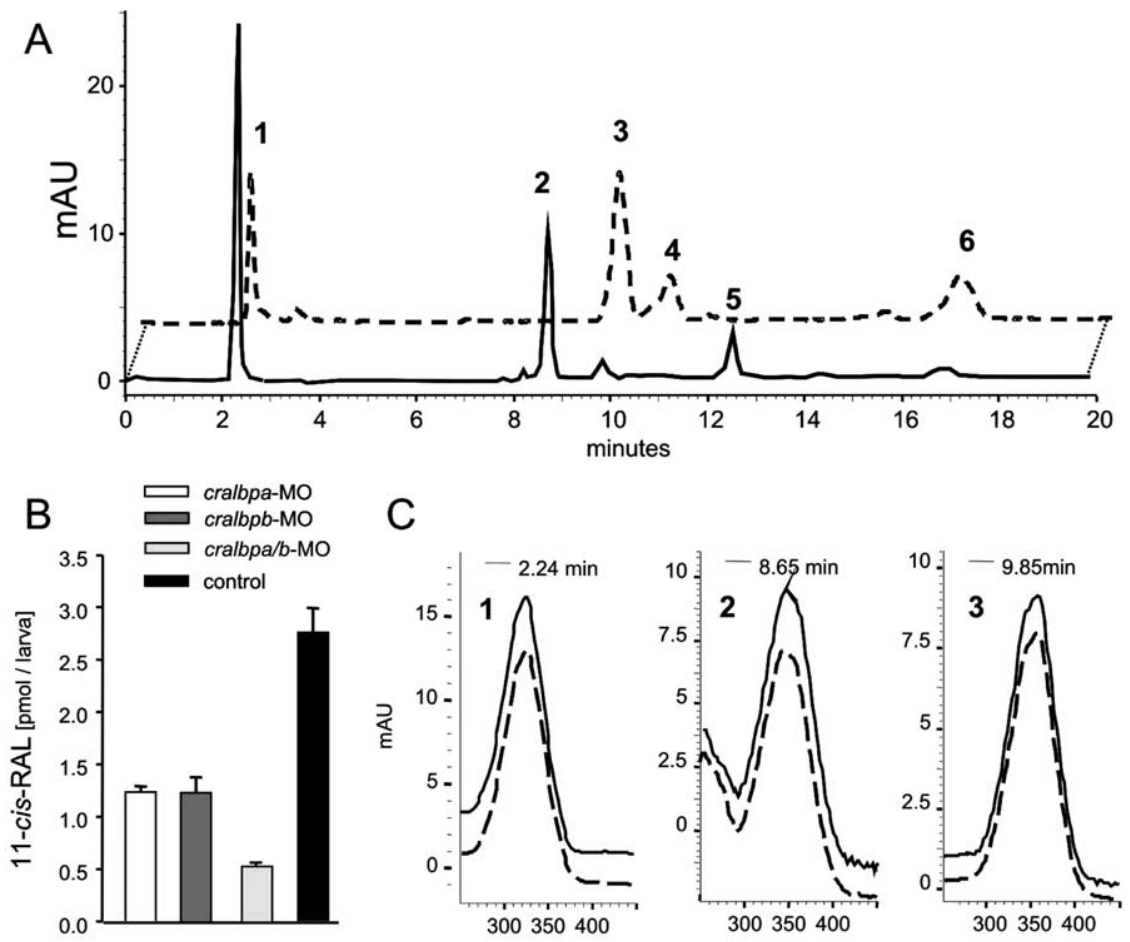

Figure 5. Levels of 11-cis-retinal in 5 dpf larvae. $A, H P L C$ traces at $325 \mathrm{~nm}$ of lipophilic extracts isolated from dark-adapted $5 \mathrm{dpf}$ larval eyes (solid line) and the remaining trunks including the yolk (dashed line). $\boldsymbol{B}, 11$-cis-Retinal levels in cralbp a, cralbp b, and co-morpholino-injected larvae compared with control larvae. All larvae were bleached (10,000 lux) and dark-readapted for 45 min before analysis. The values give the mean \pm SD of three independent experiments. $C$, Spectral characteristics of different vitamin A derivatives (solid line) compared with authentic standard substances (dashed line). 1, retinyl esters; 2, 11-cis-retinal-oxime (syn); 3, all-trans-retinal-oxime; 4, 13-cis-retinal-oxime (syn and anti), 5, 11-cis-retinal-oxime (anti); all-trans-retinal (anti).

cralbp paralogs in a cell-specific manner either in the inner retina (Cralbp b) or in the RPE (Cralbp a).

To examine the effect of reduced Cralbp levels on the integrity of the retina, standard histological sections of $5 \mathrm{dpf}$ control and morpholino-injected larvae were analyzed for retinal morphology and layering. No morphological alterations were detected in cralbp $a, b$, and double-morpholino-injected larvae, and no cell loss was visible (Fig. 4A-D). To look more closely at photoreceptor morphology, we performed immunostaining for red-green double cones using the Zpr1 (Fret43) antibody and for rods using the 1D1 antibody. Cone and rod outer segments appeared normal in all morpholino-injected larvae (Fig. $4 E-H$ ).

\section{cralbp morpholino-injected larvae have reduced levels of 11-cis-retinal}

To assess the effect of the targeted knock-down of different cralbp paralogs on ocular retinoid metabolism, we determined 11-cisretinal levels. For this purpose, $4 \mathrm{dpf}$ morpholino-injected larvae and control siblings (50 larvae per group) were raised under a standard day-and-night light regimen (14/10 h). The larvae were dark-adapted overnight before analysis. At $5 \mathrm{dpf}$, larvae were bleached by bright light (10,000 lux) for $20 \mathrm{~min}$ and were darkreadapted for $45 \mathrm{~min}$. We then determined 11-cis-retinal levels in whole larvae as a measure for the visual pigment content in the eyes. In a control experiment, we showed that 11-cis-retinal is only present in the eyes and absent in other body parts of the larva including the yolk (Fig. 5A). Importantly, 11-cis-retinal can be distinguished by its retention time and spectral characteristics from the all-trans- and 13-cis-retinal stereoisomers, which are abundant in the larval yolk (Fig. $5 A, C$ ). In repeated experiments

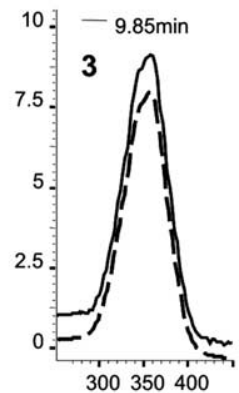

$(n=3)$, we found that the amounts of 11-cis-retinal were reduced in both cralbp $a$ and $b$ morpholino-injected larvae. Knocking-down either of the Cralbp proteins reduced the amounts of 11-cis-retinal to $\sim 50 \%$ of wild-type control levels (Fig. $5 B$ ), and coinjection of both morpholinos resulted in a further reduction of 11-cisretinal levels down to $\sim 25 \%$ of control levels (Fig. 5B). Interestingly, a comparable reduction of the 11-cis-retinal levels was reported in Rpe65-deficient zebrafish larvae devoid of visual pigment regeneration via the canonical visual cycle. Thus, we concluded that both Cralbp a in the RPE and Cralbp b in Müller cells are required for 11-cis-retinal regeneration in zebrafish.

\section{Cralbp-deficient larvae have diminished} visual performance

The electroretinogram records the sum field electrical potential of the eye and is a sensitive physiological tool to assess outer retina function in larval zebrafish.

We used a $100 \mathrm{~ms}$ light stimulus (of 5 log units of light intensities with the highest light intensity/log 0 corresponding to 6300 lux) to elicit an ERG response in $5 \mathrm{dpf}$ control and morpholino-injected larvae on dark adaption. Typical traces are depicted in Figure $6 A$, and $B$ shows a graph representing the intensity response functions. Morpholino-injected larvae showed decreased b-wave amplitudes at all light intensities compared with controls. Moreover, the intensity response function was shifted along the intensity axis (Fig. $6 A, B$ ). For a quantification of responses, we used the b-wave amplitude, which is a robust measure for outer retinal function (Rinner et al., 2005b). A quantification of saturated responses (to a light stimulus of $6000 \mathrm{lux}$ ) is depicted in Figure 6C. Under this condition, the b-wave amplitude of cralbp $a$ morpholino-injected larvae was decreased to $128.8 \mu \mathrm{V}$ compared with control larvae with a b-wave amplitude of $273.9 \mu \mathrm{V}$. In cralbp $b$ morpholino-injected larvae, the b-wave amplitude showed an even stronger decrease to $77.02 \mu \mathrm{V}$, and combined injections of both MOs resulted in a reduction of the b-wave amplitude to $57.5 \mu \mathrm{V}$. We induced a $\mathrm{MO}$ concentrationdependent decrease of the b-wave amplitude in cralbp $b$ morpholino-injected larvae, demonstrating the specificity of the measured effects in ERG analysis (data not shown).

Together, these data demonstrate that the deficiency of either Cralbp reduced light responsiveness of the larval retina. Importantly, Cralbp b deficiency of Müller cells had a more pronounced effect than Cralbp a deficiency of the RPE, indicating that it is more relevant for visual pigment regeneration in cones.

\section{Visual behavior is affected in Cralbp b-deficient larvae}

To directly assess the effects of Cralbp deficiency on visual performance, we used a vision-dependent optokinetic response test. Tracking eye movements were triggered by movement in the visual field. To compare visual performance between cralbp morpholino-injected larvae and control larvae, we measured a contrast sensitivity function. Visual performance was not af- 
fected in cralbp a morpholino-injected larvae (Fig. $7 A$ ). In contrast to that, cralbp $b$ morpholino-injected larvae showed a significantly $(p<0.05)$ decreased visual performance at higher contrast gratings (Fig. 7B). Thus, larvae suffering from Cralbp deficiency of Müller cells show an impaired visual behavior under light conditions when cones are functional.

\section{Discussion}

The photoisomerization of the chromophore 11-cis-retinal to all-trans-retinal in the photoreceptor outer segments is the first step in phototransduction. Continuous vision necessitates the regeneration of the visual chromophore in a process termed the visual cycle. The canonical visual cycle is well described for visual pigment stemming from rod photoreceptors. It is located in both photoreceptor cells and the RPE.

Recently, evidence has accumulated that in cone-dominant species another visual cycle exists that is mainly concerned with regeneration of visual pigment from cone photoreceptors. The competition for 11-cis-retinal, the visual chromophore for both rod and cone photoreceptors, might have required the evolution of rod- and cone-specific regeneration mechanisms. Both photoreceptor types work at different light intensities, so that the high demand of visual pigment for rods at photopic conditions might restrict the availability of the visual chromophore for cones in their working range (Mata et al., 2002; Muniz et al., 2007).

The existence of such a cone-specific regeneration pathway is supported by biochemical and genetic data. Earlier work implicated Müller glia cells of the inner retina in the recycling of the visual chromophore (Goldstein and Wolf, 1973; Hood and Hock, 1973; Saari et al., 1982; Bunt-Milam and Saari, 1983; Saari and Bredberg, 1987).

Work on the dynamics of retinoid accumulation in the cone-dominant chicken retina found evidence for two pools of 11-cis-retinyl esters with different depletion dynamics (Trevino et al., 2005). Genetic evidence for an alternative pathway was provided by knock-down studies of the retinoid isomerase Rpe65, a pivotal component of the canonical visual cycle. Depletion of Rpe65 in the conedominant zebrafish retina leads to decreased 11-cis-retinal levels but does not exert any effect on the larval optokinetic response, which originates exclusively in cone photoreceptors at this developmental stage (Schonthaler et al., 2007)

Mata et al. (2005) identified several novel enzymatic activities in the chicken retina that constitute likely components of an alternative cone-specific visual cycle. Consistent with earlier work, these components are suggested to be located in cone pho$b \mathrm{MO})$.
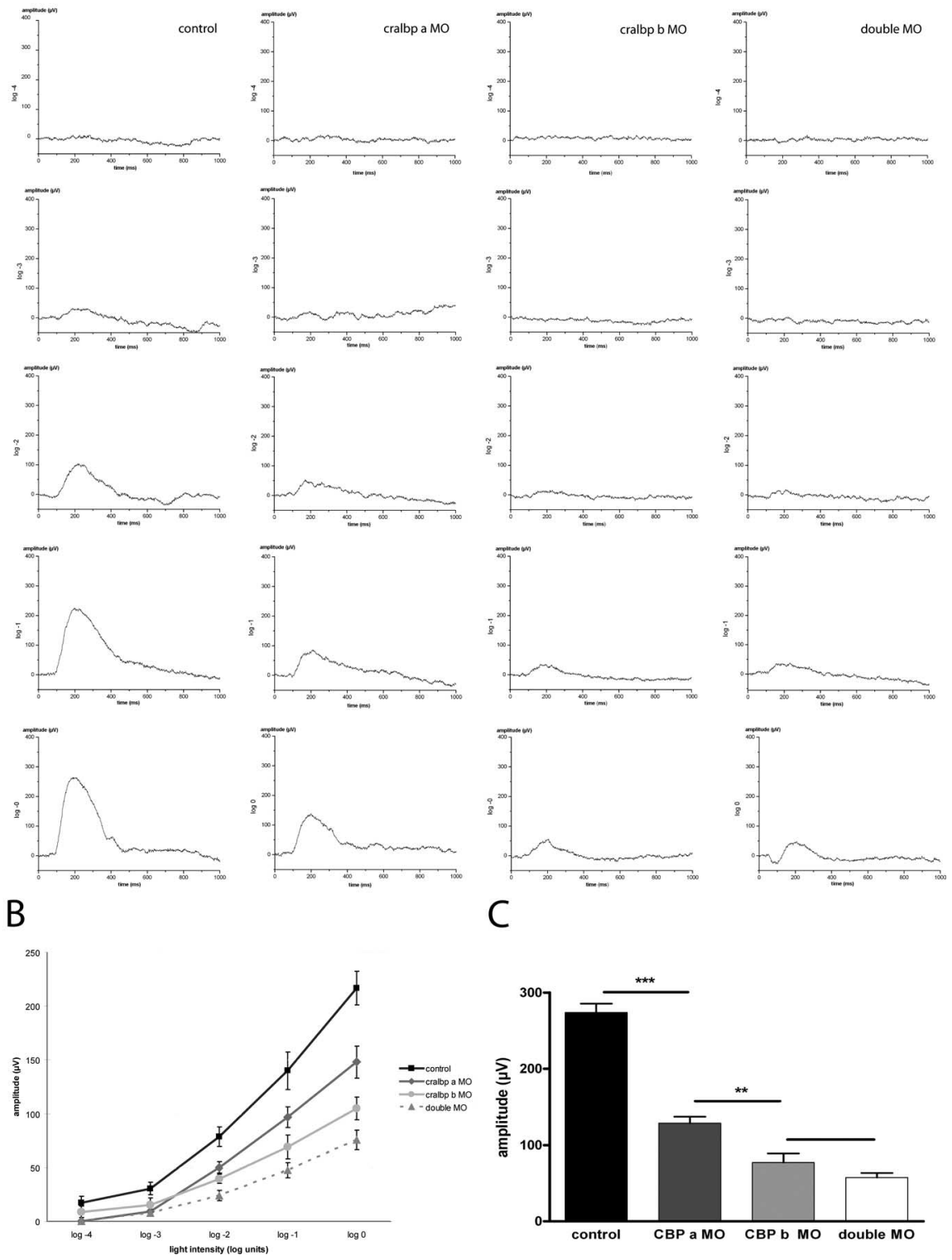

C

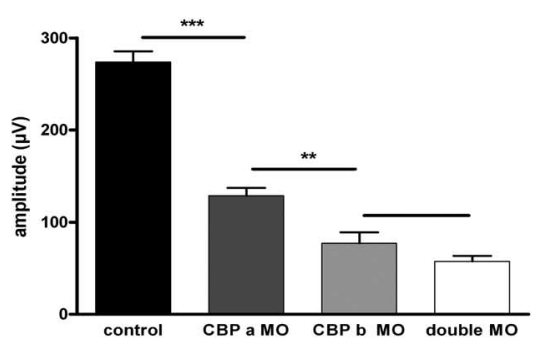

Figure 6. ERG recordings of cralbp $a, b$, and double-morpholino-injected larvae. A, Typical ERG traces of dark-adapted $5 \mathrm{dpf}$ control and morpholino-injected larvae (cralbp a, $1 \mathrm{~mm}$; cralbp b, $700 \mu \mathrm{m}$; coinjected at $800 \mu \mathrm{m} / 500 \mu \mathrm{m}$ ) at different light (log 0 corresponds to 6300 lux or $6.9 \mathrm{~mW} / \mathrm{cm}^{2}$ ). B, Graph depicting the intensity-response function of controls compared with morpholino-injected larvae (data points represent means of $n=10$ larvae for each light intensity \pm SEM). $C$ Table of averaged saturated b-wave responses (means of $n=12$ larvae \pm SD) of controls compared with morpholino-injected larvae (light intensity, 6000 lux). Statistical analysis was performed with GraphPad Prism software using one-way ANOVA on measured data ( $p<0.001$ for cralbp $a$ and $b$ and double M0 compared with wild type; $p<0.01$ for cralbp a compared with cralbp

toreceptors and Müller glia cells. None of these components has been identified yet.

To initiate a molecular characterization of these components, we used the cone-dominant zebrafish retina. We reasoned that retinoid-binding proteins might be common components to both the canonical and the alternative visual cycle.

Retinoid-binding proteins (CRBP, IRBP, CRALBP) have been shown to have a variety of different functions in the canonical visual cycle (Bridges et al., 1984; Okajima et al., 1990; Pepperberg et al., 1993; Palczewski et al., 1999). CRALBP protein specifically binds 11-cis-retinoids (Bunt-Milam and Saari, 1983; Saari and Bredberg, 1987) and has an important role in the isomerization 


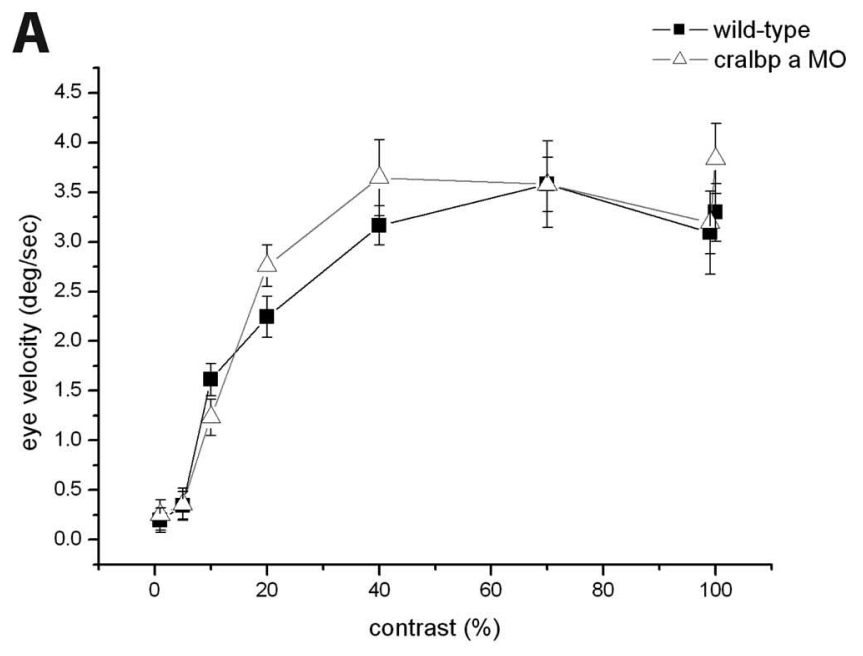

B

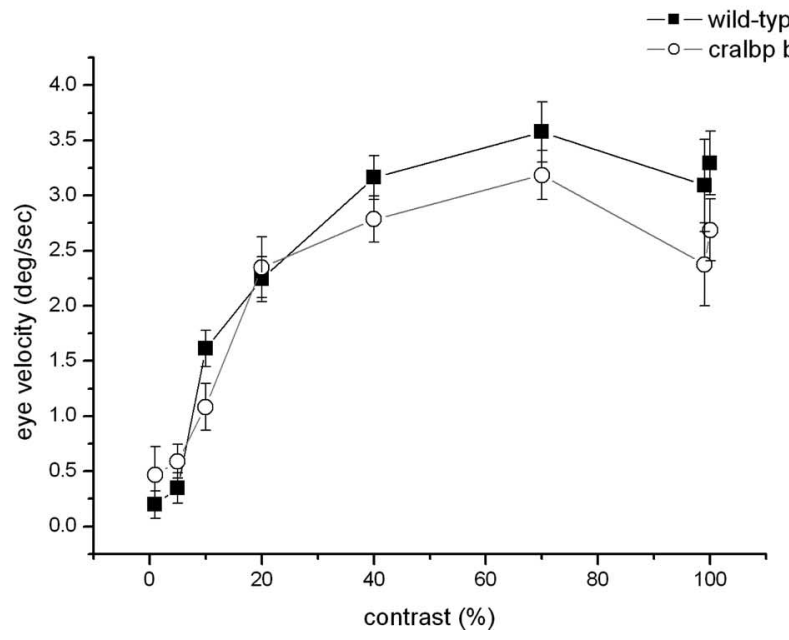

Figure 7. OKR recordings of cralbp $a$ and $b$ morpholino-injected larvae compared with wild type. OKR responses of $5 \mathrm{dpf}$ controls compared with cralbp morpholino-injected larvae triggered by gratings of varying contrast ( $0-99 \%)$. Measurements were done under dark-adapted conditions with averaged responses of $n=12$. Statistical analysis was performed using a bootstrap resampling test of pooled data; the significance level was chosen to be $5 \%$ for all recordings ( $p<0.05$ for cralbp b M0 at contrasts between 70 and $100 \%$ ). $A$, Wild-type versus cralbp a morpholino-injected larvae. $\boldsymbol{B}$, Wild-type versus cralbp $b$ morpholino-injected larvae $(p<0.05$ for contrasts $>40 \%)$.

and oxidation steps of the visual cycle (Saari and Bredberg, 1987; Saari et al., 2001; Golovleva et al., 2003).

Here, we report the identification of two paralogous cralbp genes in the zebrafish genome. The family of ray-finned (teleost) fish has undergone an additional whole-genome duplication in the course of its evolution, as compared with the other vertebrate groups (Taylor et al., 2003; Meyer and Van de Peer, 2005). As a consequence, a sizable number of genes are found in duplicate versions. The stable occurrence of such paralogs is likely caused by either neofunctionalization or subfunctionalization, because the retention of functional redundancy over the course of many millions of years is unlikely.

We found complementary expression patterns for the two zebrafish cralbp paralogs, lending strong support for a subfunctionalization event in which the function of one ortholog in two cell types is split between the paralogs. Indeed, we found one paralog expressed in Müller glia cells and the other in the RPE, enabling us to separately probe the function of this protein and its involvement in the two pathways.

We selectively depleted the proteins separately by morpholino-mediated translation blockage. Functional analysis using behavioral and electrophysiological assays of larval zebrafish devoid of either protein (Cralbp a or b), or both, revealed that a knock-down resulted in impaired vision. We have observed that loss of Cralbp b protein, which is localized in Müller glia cells, leads to more pronounced effects on photoreceptor function, as measured by ERG and OKR recordings. However, downregulation of the RPE-specific Cralbp a variant also causes a decrease in b-wave amplitude. Biochemical analysis confirmed that loss of Cralbp protein leads to reduced levels of 11-cis-retinal in photoreceptors after photobleaching.

Thereby we showed that Cralbp not only is a component of the canonical visual cycle but also functions in the cone-specific alternative visual cycle. In the canonical visual cycle, Cralbp acts downstream of Rpe65 in visual chromophore regeneration (Saari and Bredberg, 1987; Saari et al., 2001; Golovleva et al., 2003). A knock-down of Cralbp a or Rpe65 leads to a significant decrease in 11-cis-retinal regeneration after bleaching (Fig. 5) (Schonthaler et al., 2007). Interestingly, both Cralbp a- and Rpe65-deficient larvae showed no deficits in optokinetic response tests (Fig. 7) (Schonthaler et al., 2007). This finding is likely explained by the existence of an alternative visual cycle that relies on Cralbp b expression in Müller cells. Cralbp b-deficient larvae showed both a reduction of 11-cis-retinal levels and deficits in visual behavior tests. As in the canonical visual cycle, Cralbp might act downstream of a Müller cell-specific retinoid isomerase on 11-cisretinal regeneration (Mata et al., 2005). Because cone vision was not completely abolished in either Cralbp a- and Cralbp b-deficient larvae, our data argue for an involvement of both visual cycles in cone pigment regeneration. This characteristic might be explained by the fact that in Cralbp b deficiency, cones might be supplied by 11-cis-retinal produced by the canonical visual cycle. Conversely, in Cralbp a-depleted larvae, cones may consume 11-cis-retinal produced by the Müller cell-specific visual cycle. In this context, it is noteworthy that a lack of Cralbp does not completely abolish 11-cis-retinal regeneration, as evidenced by the analysis of Cralbp ${ }^{-1-}$ mice. This characteristic might also explain the persisting 11-cis-retinal in the larvae depleted for both Cralbp paralogs. In future studies, the relative importance of the canonical and alternative visual cycle for cone visual pigment regeneration needs to be established. It likely depends on the species-specific photoreceptor composition and arrangement of the outer retina. We expect that the reliance of cone pigment regeneration on the non-canonical pathway will increase with the increase in rod contribution to zebrafish vision occurring at later larval stages (Branchek, 1984). Our data are in full agreement with a recent study conducted in patients suffering from Leber congenital amaurosis. Such patients carry a mutated version of the retinoid isomerase RPE65 and, as a consequence, they exhibit cone photoreceptor loss even at the earliest stages of disease. Strikingly, this loss is not complete, and residual cone structure and function persist for decades (Jacobson et al., 2007). We conclude that the RPE65-based visual cycle must be important for cone function, but there must also exist an alternative pathway for visual pigment regeneration, which could explain cone survival.

In summary, we have identified the first molecular component of the cone-specific alternative visual cycle, using the favorable genetics of the cone-dominant zebrafish. The two paralogs of the retinoid-binding protein Cralbp play a role in two separate 
visual pigment regeneration pathways, as evidenced by biochemical, genetic, physiological, and behavioral data. These visual cycles are located in two separate compartments in the zebrafish retina: the canonical visual cycle in the RPE and the proposed cone-specific cycle in Müller glia cells. Because interfering with both pathways leads to functional deficits in the cone-dominant retina of the zebrafish, we propose that cone photoreceptors use both mechanisms of pigment regeneration. Both visual cycles depend on the function of the retinoid-binding protein Cralbp.

\section{References}

Branchek T (1984) The development of photoreceptors in the zebrafish, brachydanio rerio. II. Function. J Comp Neurol 224:116-122.

Bridges CD, Alvarez RA, Fong SL, Gonzalez-Fernandez F, Lam DM, Liou GI (1984) Visual cycle in the mammalian eye. Retinoid-binding proteins and the distribution of 11-cis retinoids. Vision Res 24:1581-1594.

Bunt-Milam AH, Saari JC (1983) Immunocytochemical localization of two retinoid-binding proteins in vertebrate retina. J Cell Biol 97:703-712.

Dahm R, Geisler, R, Nüsslein-Volhard C (2005) Zebrafish (Danio rerio) genome and genetics. Weinheim, Germany: Wiley-VCH.

Goldstein EB, Wolf BM (1973) Regeneration of the green-rod pigment in the isolated frog retina. Vision Res 13:527-534.

Golovleva I, Bhattacharya S, Wu Z, Shaw N, Yang Y, Andrabi K, West KA, Burstedt MS, Forsman K, Holmgren G, Sandgren O, Noy N, Qin J, Crabb JW (2003) Disease-causing mutations in the cellular retinaldehyde binding protein tighten and abolish ligand interactions. J Biol Chem 278:12397-12402.

Hood DC, Hock PA (1973) Recovery of cone receptor activity in the frog's isolated retina. Vision Res 13:1943-1951.

Jacobson SG, Aleman TS, Cideciyan AV, Heon E, Golczak M, Beltran WA, Sumaroka A, Schwartz SB, Roman AJ, Windsor EA, Wilson JM, Aguirre GD, Stone EM, Palczewski K (2007) Human cone photoreceptor dependence on RPE65 isomerase. Proc Natl Acad Sci U S A 104:15123-15128.

Jin M, Li S, Moghrabi WN, Sun H, Travis GH (2005) Rpe65 is the retinoid isomerase in bovine retinal pigment epithelium. Cell 122:449-459.

Makhankov YV, Rinner O, Neuhauss SC (2004) An inexpensive device for non-invasive electroretinography in small aquatic vertebrates. J Neurosci Methods 135:205-210.

Mata NL, Radu RA, Clemmons RC, Travis GH (2002) Isomerization and oxidation of vitamin a in cone-dominant retinas: a novel pathway for visual-pigment regeneration in daylight. Neuron 36:69-80.

Mata NL, Ruiz A, Radu RA, Bui TV, Travis GH (2005) Chicken retinas contain a retinoid isomerase activity that catalyzes the direct conversion of all-trans-retinol to 11-cis-retinol. Biochemistry 44:11715-11721.

McBee JK, Van Hooser JP, Jang GF, Palczewski K (2001) Isomerization of 11-cis-retinoids to all-trans-retinoids in vitro and in vivo. J Biol Chem 276:48483-48493.

Meyer A, Van de Peer Y (2005) From 2R to 3R: evidence for a fish-specific genome duplication (FSGD). Bioessays 27:937-945.

Moiseyev G, Chen Y, Takahashi Y, Wu BX, Ma JX (2005) RPE65 is the isomerohydrolase in the retinoid visual cycle. Proc Natl Acad Sci U S A 102:12413-12418.

Muniz A, Villazana-Espinoza ET, Hatch AL, Trevino SG, Allen DM, Tsin AT (2007) A novel cone visual cycle in the cone-dominated retina. Exp Eye Res 85:175-184.

Nasevicius A, Ekker SC (2000) Effective targeted gene 'knockdown' in zebrafish. Nat Genet 26:216-220.
Okajima TI, Pepperberg DR, Ripps H, Wiggert B, Chader GJ (1990) Interphotoreceptor retinoid-binding protein promotes rhodopsin regeneration in toad photoreceptors. Proc Natl Acad Sci U S A 87:6907-6911.

Palczewski K, Van Hooser JP, Garwin GG, Chen J, Liou GI, Saari JC (1999) Kinetics of visual pigment regeneration in excised mouse eyes and in mice with a targeted disruption of the gene encoding interphotoreceptor retinoid-binding protein or arrestin. Biochemistry 38:12012-12019.

Palczewski K, Hofmann KP, Baehr W (2006) Rhodopsin-advances and perspectives. Vision Res 46:4425-4426.

Pepperberg DR, Okajima TL, Wiggert B, Ripps H, Crouch RK, Chader GJ (1993) Interphotoreceptor retinoid-binding protein (IRBP). Molecular biology and physiological role in the visual cycle of rhodopsin. Mol Neurobiol 7:61-85.

Redmond TM, Poliakov E, Yu S, Tsai JY, Lu Z, Gentleman S (2005) Mutation of key residues of RPE65 abolishes its enzymatic role as isomerohydrolase in the visual cycle. Proc Natl Acad Sci U S A 102:13658-13663.

Rinner O, Rick JM, Neuhauss SC (2005a) Contrast sensitivity, spatial and temporal tuning of the larval zebrafish optokinetic response. Invest Ophthalmol Vis Sci 46:137-142.

Rinner O, Makhankov YV, Biehlmaier O, Neuhauss SC (2005b) Knockdown of cone-specific kinase GRK7 in larval zebrafish leads to impaired cone response recovery and delayed dark adaptation. Neuron 47:231-242.

Saari JC, Bredberg DL (1987) Photochemistry and stereoselectivity of cellular retinaldehyde-binding protein from bovine retina. J Biol Chem 262:7618-7622.

Saari JC, Bredberg L, Garwin GG (1982) Identification of the endogenous retinoids associated with three cellular retinoid-binding proteins from bovine retina and retinal pigment epithelium. J Biol Chem 257:13329-13333.

Saari JC, Nawrot M, Kennedy BN, Garwin GG, Hurley JB, Huang J, Possin DE, Crabb JW (2001) Visual cycle impairment in cellular retinaldehyde binding protein (CRALBP) knockout mice results in delayed dark adaptation. Neuron 29:739-748.

Schoenlein RW, Peteanu LA, Mathies RA, Shank CV (1991) The first step in vision: femtosecond isomerization of rhodopsin. Science 254:412-415.

Schonthaler HB, Lampert JM, Isken A, Rinner O, Mader A, Gesemann M, Oberhauser V, Golczak M, Biehlmaier O, Palczewski K, Neuhauss SC, von Lintig J (2007) Evidence for RPE65-independent vision in the conedominated zebrafish retina. Eur J Neurosci 26:1940-1949.

Stecher H, Gelb MH, Saari JC, Palczewski K (1999) Preferential release of 11-cis-retinol from retinal pigment epithelial cells in the presence of cellular retinaldehyde-binding protein. J Biol Chem 274:8577-8585.

Stryer L (1986) Cyclic GMP cascade of vision. Annu Rev Neurosci 9:87-119.

Taylor JS, Braasch I, Frickey T, Meyer A, Van de Peer Y (2003) Genome duplication, a trait shared by 22000 species of ray-finned fish. Genome Res 13:382-390.

Trevino SG, Villazana-Espinoza ET, Muniz A, Tsin AT (2005) Retinoid cycles in the cone-dominated chicken retina. J Exp Biol 208:4151-4157.

von Lintig J, Vogt K (2000) Filling the gap in vitamin A research. Molecular identification of an enzyme cleaving beta-carotene to retinal. J Biol Chem 275:11915-11920.

Wald G (1968) The molecular basis of visual excitation. Nature 219:800-807.

Westerfield M (1994) The zebrafish book: a guide for the laboratory use of zebrafish (Danio rerio). Eugene, OR: University of Oregon.

Winston A, Rando RR (1998) Regulation of isomerohydrolase activity in the visual cycle. Biochemistry 37:2044-2050. 